\section{Prevalencia y características de mutaciones somáticas del gen KRAS en pacientes chilenos con cáncer colorrectal}

\author{
CLAUDIA HURTADO ${ }^{1, \mathrm{a}}$, GONZALO ENCINA ${ }^{1, \mathrm{a}}$, ANA MARÍA WIELANDT ${ }^{1, \mathrm{~b}}$, \\ ALEJANDRO JOSÉ ZÁRATE ${ }^{1}$, MAGDALENA CASTRO ${ }^{3, c}$, \\ KATYA CARRILLO $^{1, \mathrm{~d}}$, UDO KRONBERG ${ }^{1}$, FRANCISCO LÓPEZ-KÖSTNER ${ }^{1}$
}

\section{KRAS gene somatic mutations in Chilean patients with colorectal cancer}

Background: The molecular testing of KRAS mutation status in metastatic colorectal cancer patients is mandatory to identify patients eligible for antiepidermal growth factor receptor monoclonal antibody therapy. Aim: To report the frequency of KRAS gene mutations in Chilean patients with colorectal cancer (CRC). Material and Methods: A cohort of 262 Chilean patients with CRC aged 26 to 90 years (53\% males), was studied. KRAS mutation status was analyzed by real-time polymerase chain reaction and correlated with clinicopathological data. Results: Ninety-eight patients (37\%) were positive for KRAS mutations. G12D was the most common mutation with a frequency of $36.7 \%$, followed by G12V (25.5\%), G13D (17.3\%), G12A (7.1\%), G12C (6.1\%), G12S (5.1\%) and $G 12 R(2 \%)$. The frequency of the mutation in left, right colon and rectal tumors was 37.8, 32.6 and 44.9\%, respectively. Among tumors with mutations, $86.7 \%$ were well or moderately differentiated tumors and the rest were poorly differentiated. No significant associations between KRAS gene mutations and other clinicopathological features of the tumor were observed. Conclusions: The frequencies of KRAS mutations reported in this study are similar to frequencies reported for European and North-American populations, lower than in a Spanish study and higher than in a Peruvian study.

(Rev Med Chile 2014; 142: 1407-1414)

Key words: KRAS protein, human; Colorectal neoplasms; Proto-oncogene proteins; Receptor, epidermal growth factor.

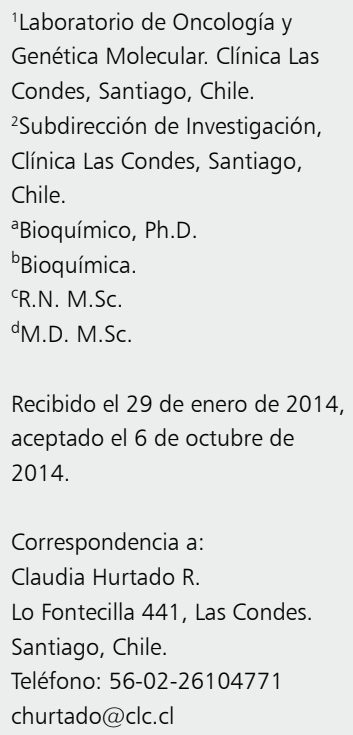

K RAS es un proto-oncogén, miembro de la familia de genes RAS que codifica para una proteína GTPasa del mismo nombre. La proteína KRAS es uno de los componentes de la red de señalización intracelular del receptor del factor de crecimiento epidermal (EGFR) que regula funciones celulares clave tales como proliferación, diferenciación y apoptosis ${ }^{1,2}$. Aproximadamente $40 \%$ de los carcinomas colorrectales (CCR) presentan mutaciones activantes en el gen KRAS.
Estas mutaciones alteran la actividad GTPasa intrínseca de la proteína KRAS, causando la acumulación de KRAS en un estado unido a GTP y permanentemente activo. De esta forma, la vía del EGFR se independiza de la unión del ligando EGF a su receptor, lo que enciende constitutivamente la cascada de señalización celular, iniciándose una respuesta proliferativa desregulada y constitutivamente activa, evento clave en la patogénesis del $\mathrm{CCR}^{3}$. Casi $90 \%$ de estas mutaciones se presentan 
en los codones 12 y 13 del exón 2 (o exón 1 codificante) del gen KRAS, siendo G12D, G12A, G12R, G12C, G12S, G12V y G13D las mutaciones más comunes, donde la glicina de los codones 12 y 13 es remplazada por ácido aspártico (D), alanina (A), arginina (R), cisteína (C), serina (S) y valina (V) respectivamente ${ }^{2,4}$. La importancia de KRAS como marcador en la toma de decisiones terapéuticas está bien establecida, ya que los pacientes con CCR metastásico (CCRm) que portan mutaciones en dichos codones no se benefician de las terapias biológicas con anticuerpos monoclonales dirigidas a inhibir al receptor EGFR, tales como cetuximab o panitumumab. De esta forma, la evaluación del estado mutacional del gen KRAS es mandatoria en pacientes con CCRm para asegurar un tratamiento adecuado, identificando aquellos pacientes no respondedores al tratamiento anti-EGFR ${ }^{5,6}$. Sin embargo, en cuanto al valor pronóstico de KRAS, pese a que existen varios estudios, no se ha consensuado que los pacientes portadores de mutaciones activantes en el gen KRAS presenten una sobrevida global y sobrevida libre de enfermedad mayor o menor respecto a aquellos pacientes que no portan dichas mutaciones ${ }^{7,8}$.

Los estudios de mutaciones en el gen KRAS se han enfocado principalmente en poblaciones europeas y de América del Norte ${ }^{2,9-12}$. Sólo un número reducido de estudios han reportado la frecuencia de mutaciones en el gen KRAS en pacientes con CCR de América Latina. Un estudio multicéntrico realizado en pacientes con CCR de Argentina, Brasil, México y Venezuela reportó una frecuencia de mutaciones en el gen KRAS de $36 \%{ }^{13}$, similar a la frecuencia reportada en Europa (40\%), pero superior a la de Asia (22\%). Un estudio realizado en 90 pacientes peruanos con CCR reportó una frecuencia de mutaciones en el gen KRAS de $16,7 \%$, identificando una nueva mutación $(\mathrm{G} 13 \mathrm{R})^{14}$.

Chile es un país en vías de desarrollo, con una población mixta, multi-étnica, con una fuerte base genética amerindia y española, pero diferente de otros países de la región. Distintos grupos étnicos presentan diferencias en la incidencia de $\mathrm{CCR}^{15}$, probablemente debido a la combinación de factores genéticos, epigenéticos, y medio ambientales. Por otra parte, un informe reciente de Zárate y cols. demostró que en los últimos 26 años Chile ha duplicado la mortalidad por CCR, alcanzando el cuarto y quinto lugar de mortalidad por cáncer en hombres y mujeres, respectivamente ${ }^{16}$. Este aumento de la mortalidad puede explicarse en parte por el aumento de la incidencia de CCR, una mayor expectativa de vida y el aumento de los factores de riesgo asociados a una occidentalización progresiva del estilo de vida como dietas rica en grasas y sedentarismo entre otros ${ }^{17}$. Muy recientemente, se reportó el primer estudio chileno de frecuencia y distribución de las mutaciones del gen KRAS en 109 muestras de cáncer de colon y recto $^{18}$, con $42 \%$ de los pacientes con KRAS mutado. El presente trabajo correlaciona los datos clínicos del paciente e histopatológicos de los tumores, con la distribución y las frecuencias de mutaciones somáticas del exón 2 del gen KRAS en una cohorte de 262 pacientes chilenos con CCR, mediante el uso de una técnica altamente sensible como es la reacción en cadena de la polimerasa (PCR) en tiempo real.

\section{Material y Método}

\section{Pacientes y muestras}

Entre octubre de 2009 y marzo de 2012 se analizaron 475 pacientes con CCR de forma prospectiva y consecutiva para el estudio de las mutaciones del exón 2 del gen KRAS a partir de ADN tumoral. Los pacientes fueron derivados de diferentes centros de salud de todo el país al Laboratorio de Oncología y Genética Molecular de Clínica Las Condes. Todos los pacientes firmaron un consentimiento informado de acuerdo con las directrices institucionales y el Comité de Ética de Clínica Las Condes. De los 475 pacientes analizados, se seleccionaron para este estudio 262 cuya información clínica estaba más completa. No obstante, en este grupo de pacientes no se pudo estimar el estadio debido a la falta de información sobre el estado metastásico a distancia de cada paciente. Los datos clínicos registrados incluyen género, edad, localización del tumor, profundidad de la invasión y compromiso linfonodal, patrón histológico y grado de diferenciación, en la medida que esta información estaba disponible en los informes de biopsias.

\section{Extracción de ADN tumoral}

El ADN tumoral se extrajo de muestras quirúrgicas fijadas en formalina e incluidas en parafina (FFPE). Brevemente, los cortes teñidos con hematoxilina-eosina fueron analizados por 
el patólogo institucional, quien seleccionó zonas enriquecidas con más de $80 \%$ de células tumorales. Se extrajo el ADN tumoral de cortes seriados de $8 \mu \mathrm{m}$ de espesor no teñidos mediante el sistema QIAmp DNA FFPE Tissue extraction kit (QIAGEN, Hilden, Alemania), según las instrucciones del fabricante. La integridad del ADN se analizó mediante la amplificación por PCR multiplex de 2 amplicones de GAPDH, 1 amplicón de $\beta$-globina y 1 amplicón de $\beta$-actina ${ }^{19}$.

\section{Análisis de mutaciones somáticas en el gen KRAS}

Las siete mutaciones somáticas más comunes en el oncogén KRAS se detectaron mediante PCR en tiempo real, utilizando el sistema de diagnóstico in vitro DxS KRAS mutations TheraScreen ${ }^{\circledR}$ de ROCHE, en un termociclador LightCycler 480 II, según las instrucciones del fabricante. El sistema de detección es un sistema validado (IVD) y altamente sensible para la detección de mutaciones específicas en el gen KRAS humano.

\section{Análisis estadístico}

Los datos categóricos se resumieron en frecuencias y porcentajes. Las asociaciones entre los datos categóricos se analizaron mediante pruebas de $\chi^{2}$. Valores de $\mathrm{p}$ inferiores a 0,05 se consideraron estadísticamente significativos.

\section{Resultados}

En el período descrito se analizaron 475 pacientes chilenos con CCR, donde 167 presentaron alguna mutación en KRAS y solamente en 4 casos el estudio fue no concluyente, lo que equivale a un hallazgo de mutaciones de $35 \%$.

De los 262 pacientes con CCR seleccionados para este análisis, $53 \%$ fueron varones, con una edad media de 61 años (26-81) al momento del diagnóstico. La edad media al momento del diagnóstico de los pacientes de género femenino fue de 63 años (28-90). El 48,5\% de los pacientes presentó cáncer de colon izquierdo, 32,8\% presentó cáncer de colon derecho y $18,7 \%$ de los pacientes presentó cáncer de recto.

Al analizar la profundidad de la invasión de los tumores de pacientes referidos a estudio se observó que presentaban una profundidad de invasión que variaba de pT1 a pT4, siendo la más frecuente pT3. También se analizaron pacientes sin compromiso linfonodal. Respecto del compromiso linfonodal, existe $70 \%$ aproximadamente de infiltración tumoral linfonodal, tanto en hombres como en mujeres.

En cuanto a patrón histológico y al grado de diferenciación, la mayoría de los tumores analizados eran de tipo tubular moderadamente diferenciado. En algunos casos se observaron tumores mucinosos o en anillo de sello y casos puntuales de tipo cribiforme, además de un tumor definido como sólido solamente. No se observó asociación entre el grado de diferenciación o patrón histológico con la presencia de mutaciones en el exón 2 de KRAS (datos no mostrados). Las características clínicas e histopatológicas de los pacientes, se presentan en la Tabla 1.

El 37\% de los pacientes (98) presentó mutaciones en el exón 2 de KRAS (Figura 1A). De 127 pacientes con cáncer de colon izquierdo, 48 $(37,8 \%)$ presentaron mutaciones en KRAS. De los 86 pacientes con cáncer de colon derecho y 49 con cáncer de recto, 28 (32,6\%) y 22 (44,9\%) pacientes presentaron mutaciones en KRAS, respectivamente (Figura 1B). El 91\% de los pacientes (239) presentó CCR con invasión hasta pT3 o pT4, siendo $36,8 \%$ de ellos positivo para mutaciones en KRAS, mientras que $43,5 \%$ de los pacientes con CCR con invasión pT1 o pT2 fueron positivos para mutación en KRAS. Los datos de mutaciones en KRAS en relación a la profundidad de invasión, los grados de diferenciación tumoral y el estado de los ganglios linfáticos se presentan en la Tabla 2. No se observaron diferencias significativas en las características clínicas e histopatológicas del tumor de pacientes con mutación en KRAS versus sin mutación.

De las 7 mutaciones de KRAS más frecuentes, G12D fue la más prevalente en los pacientes estudiados con 36,7\%, seguido de G12V, G13D, G12A, G12C, G12S y, finalmente, G12R. En relación con el tipo de mutaciones de KRAS identificadas y la edad de diagnóstico de CCR, la mutación G12C pareciera estar presente en tumores con diagnóstico a edades más tempranas (60,5 años) y la mutación G12S más prevalente en edades avanzadas (69 años), sin embargo, estos resultados no tienen una diferencia estadísticamente significativa (Tabla 3). Tampoco se observaron diferencias significativas entre el tipo de mutaciones del gen KRAS y género (datos no mostrados). 
Tabla 1. Características clínicas e histopatológicas de pacientes chilenos con cáncer colorrectal

\begin{tabular}{|c|c|c|c|}
\hline & Total & Hombres & Mujeres \\
\hline Pacientes & 262 & $139(53 \%)$ & $123(47 \%)$ \\
\hline Edad al diagnóstico (rango) & $62(26-90)$ & $61(26-81)$ & $63(28-90)$ \\
\hline $\begin{array}{l}\text { Localización tumoral } \\
\text { Colon derecho } \\
\text { Colon izquierdo } \\
\text { Recto }\end{array}$ & $\begin{array}{rr}86 & (32,8 \%) \\
127 & (48,5 \%) \\
49 & (18,7 \%)\end{array}$ & $\begin{array}{l}49(35,3 \%) \\
67(48,2 \%) \\
23(16,5 \%)\end{array}$ & $\begin{array}{ll}37 & (30,1 \%) \\
60 & (48,8 \%) \\
26 & (21,1 \%)\end{array}$ \\
\hline $\begin{array}{l}\text { Profundidad de invasión } \\
\text { PT1 } \\
\text { pT2 } \\
\text { pT3 } \\
\text { pT4 }\end{array}$ & $\begin{array}{rr}1 & (0,4 \%) \\
22 & (8,4 \%) \\
179 & (68,3 \%) \\
60 & (22,9 \%)\end{array}$ & $\begin{aligned} 0 & (0 \%) \\
14 & (10,1 \%) \\
89 & (64 \%) \\
36 & (25,9 \%)\end{aligned}$ & $\begin{array}{rr}1 & (100 \%) \\
8 & (6,5 \%) \\
90 & (73,2 \%) \\
24 & (19,5 \%)\end{array}$ \\
\hline $\begin{array}{l}\text { Estado linfonodal } \\
\qquad \begin{array}{l}\mathrm{NO} \\
\mathrm{N}+\end{array}\end{array}$ & $\begin{array}{rr}72 & (27,5 \%) \\
190 & (72,5 \%)\end{array}$ & $\begin{array}{rr}39 & (28,1 \%) \\
100 & (71,9 \%)\end{array}$ & $\begin{array}{ll}33 & (26,8 \%) \\
90 & (73,2 \%)\end{array}$ \\
\hline $\begin{array}{l}\text { Patrón histológico } \\
\text { Tubular } \\
\text { Mucinoso } \\
\text { Anillo de sello } \\
\text { Cribriforme } \\
\text { Sólido }\end{array}$ & $\begin{aligned} 231 & (88,1 \%) \\
21 & (8 \%) \\
7 & (2,7 \%) \\
2 & (0,8 \%) \\
1 & (0,4 \%)\end{aligned}$ & $\begin{array}{rr}119 & (85,6 \%) \\
13 & (9,4 \%) \\
5 & (3,6 \%) \\
1 & (0,7 \%) \\
1 & (0,7 \%)\end{array}$ & $\begin{aligned} 112 & (91,1 \%) \\
8 & (6,5 \%) \\
2 & (1,6 \%) \\
1 & (0,8 \%) \\
0 & (0 \%)\end{aligned}$ \\
\hline $\begin{array}{l}\text { Grado de diferenciación } \\
\text { Bien diferenciado } \\
\text { Moderadamente diferenciado } \\
\text { Pobremente diferenciado }\end{array}$ & $\begin{aligned} 37 & (14,1 \%) \\
175 & (66,8 \%) \\
50 & (19,1 \%)\end{aligned}$ & $\begin{array}{ll}22 & (15,8 \%) \\
88 & (63,3 \%) \\
29 & (20,9 \%)\end{array}$ & $\begin{array}{ll}15 & (12,2 \%) \\
87 & (70,7 \%) \\
21 & (17,1 \%)\end{array}$ \\
\hline
\end{tabular}

* Las diferencias no fueron estadísticamente significativas, Valores de $p>0,05$
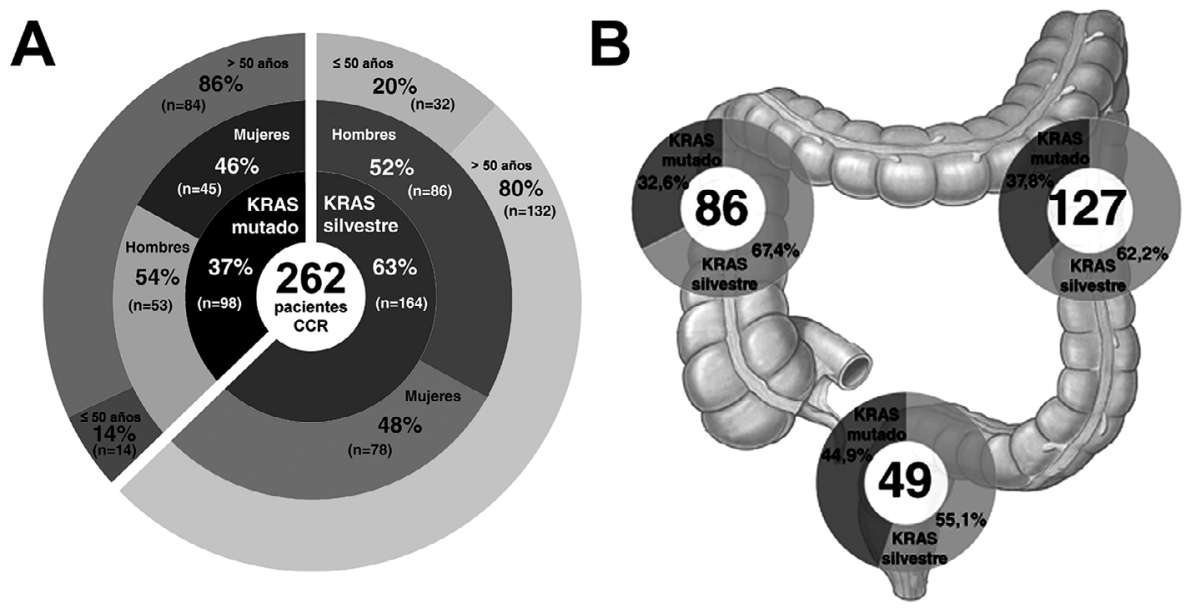

Figura 1. Distribución del estado mutacional del gen KRAS según género, edad y localización tumoral en pacientes chilenos con CCR. Se estudió el estado mutacional del gen KRAS en tumores de 262 pacientes chilenos con CCR que presentaban datos clínicos e histopatológicos completos. (A) Distribución de pacientes con KRAS mutado y KRAS silvestre según género y rango etario. (B) Distribución de pacientes con KRAS mutado y KRAS silvestre según la localización del tumor, dividido en colon derecho, colon izquierdo y recto. 
Mutaciones somáticas del gen KRAS en pacientes chilenos con cáncer colorrectal - C. Hurtado et al

Tabla 2. Mutaciones en KRAS según profundidad de invasión tumoral, diferenciación y estado linfonodal

\begin{tabular}{|lcccccc|}
\hline Pacientes & \multicolumn{2}{c}{$\begin{array}{c}\text { Invasión tumoral } \\
\text { pT1-pT2 }\end{array}$} & pT3-pT4 & $\begin{array}{c}\text { Grado de diferenciación } \\
\text { Bien/moderado }\end{array}$ & $\begin{array}{c}\text { Estado linfonodal } \\
\text { Pobre }\end{array}$ & N+ \\
$\begin{array}{l}\text { Total } \\
n=262\end{array}$ & 23 & 239 & 212 & 50 & 190 & 72 \\
$\begin{array}{l}\text { KRAS sin mutación } \\
n=164\end{array}$ & $13(56,5 \%)$ & $151(63,2 \%)$ & $127(60 \%)$ & $37(74 \%)$ & $117(61,6 \%)$ & $47(65,3 \%)$ \\
$\begin{array}{l}\text { KRAS mutado } \\
n=98\end{array}$ & $10(43,5 \%)$ & $88(36,8 \%)$ & $85(40 \%)$ & $13(26 \%)$ & $73(38,4 \%)$ & $25(34,7 \%)$ \\
\hline
\end{tabular}

* Las diferencias no fueron estadísticamente significativas. Valores de $p>0,05$

Tabla 3. Distribución del tipo de mutaciones en el exón 2 de KRAS

\begin{tabular}{|lllllcl|}
\hline $\begin{array}{l}\text { Mutación } \\
\text { en KRAS }\end{array}$ & Total & $\begin{array}{l}\text { Colon } \\
\text { derecho }\end{array}$ & $\begin{array}{c}\text { Colon } \\
\text { izquierdo }\end{array}$ & Recto & $\begin{array}{c}\text { Edad al diagnóstico } \\
\text { (mediana) }\end{array}$ & p \\
\hline Todas & 98 & 28 & 48 & 22 & 62 & NS* \\
G12D & $36(36,7 \%)$ & $10(35,7 \%)$ & $17(35,4 \%)$ & $9(40,9 \%)$ & 62,5 & NS* \\
G12V & $25(25,5 \%)$ & $7(25 \%)$ & $12(25 \%)$ & $6(27,3 \%)$ & 64 & NS* \\
G13D & $17(17,3 \%)$ & $8(28,6 \%)$ & $6(12,5 \%)$ & $3(13,6 \%)$ & 64 & NS* \\
G12A & $7(7,1 \%)$ & 0 & $6(12,5 \%)$ & $1(4,5 \%)$ & 61 & NS* \\
G12C & $6(6,1 \%)$ & 0 & $4(8,3 \%)$ & $2(9,0 \%)$ & 60,5 & NS* \\
G12S & $5(5,1 \%)$ & $3(10,7 \%)$ & $2(4,2 \%)$ & 0 & 69 & NS* \\
G12R & $2(2 \%)$ & 0 & $1(2,0 \%)$ & $1(4,5 \%)$ & 66 & NS* \\
Sin mutación & 164 & 58 & 79 & 27 & 61 & NS* \\
\hline
\end{tabular}

NS*: Las diferencias no fueron estadísticamente significativas, Valores de $p>0,05$

\section{Discusión}

$\mathrm{Al}$ considerar los 475 pacientes chilenos con CCR analizados inicialmente en este estudio, se observó que $35 \%$ presentaron mutaciones activantes en los codones 12 y 13 del gen KRAS; este valor varía levemente a 37,4\% cuando se consideran sólo los 262 pacientes con datos clínico-patológicos completos. Ambos valores caen dentro del rango descrito en estudios realizados en América del Norte y Europa ${ }^{2,4,9-12,20,21}$. Un estudio similar realizado recientemente en una cohorte de 109 pacientes chilenos con CCR reportó $42 \%$ de mutación en $\mathrm{KRAS}^{18}$, similar a $48 \%$ reportado por un estudio español realizado en una cohorte de 186 pacientes $^{22}$. Por otra parte, un estudio realizado en una cohorte de 90 pacientes peruanos con CCR metastásico reportó una frecuencia de mutación en KRAS de $16,7 \%{ }^{14}$. Se estima que la población indígena en el Perú bordea 30\%-45\% del total nacional ${ }^{14,23}$. La población chilena tiene una estructura étnica conformada por $5 \%$ de indígenas, $30 \%$ de blancos o caucásicos y $65 \%$ de mestizos predominantemente blancos ${ }^{24}$. El aporte caucásico proviene fundamentalmente de españoles, lo que explicaría en parte la mayor similitud en las frecuencias de mutaciones reportadas por el estudio español ${ }^{22}$. Por otra parte, el mayor componente genético amerindio del Perú podría dar cuenta de la menor frecuencia de mutaciones observada en la población peruana respecto a la chilena y española. En nuestro grupo de estudio no determinamos la composición amerindia para explicar la similitud de la frecuencia de mutaciones en KRAS con otras poblaciones, pero ello se podría hacer analizando en nuestros pacientes cuatro haplotipos fundadores de ADN mitocondrial para poblaciones amerindias que están bien establecidos ${ }^{24}$. 
Los codones 12 y 13 en el exón 2 del gen KRAS codifican para el aminoácido glicina $(G)$ en la proteína nativa. Nuestros resultados muestran semejanzas con otros estudios ${ }^{6,19}$, siendo G12D, G12V y G13D las mutaciones más frecuentes con $36 \%, 25 \%$ y $17 \%$, respectivamente. Mutaciones en estos codones son las principales responsables de la activación constitutiva de la proteína, y de la independización de la vía del EGFR por su ligando ${ }^{3,25}$.

Sin embargo, se han descrito otras mutaciones en el exón 2 (codones 22 y 31 ,en los exones 3 (codón 61) y 4 (codones 117 y 146) del gen KRAS, que tienen de la misma manera un efecto activante en la proteína ${ }^{26-28}$. Aunque estas se encuentran en una menor proporción, son también relevantes al momento de determinar si terapias biológicas como cetuximab o panitumumab pueden ser indicadas en un paciente dado, ya que igualmente explican la falta de respuesta a estas terapias ${ }^{29}$.

La detección de mutaciones en este estudio se realizó por PCR en tiempo real mediante un sistema de diagnóstico validado, altamente sensible que detecta las 7 mutaciones más comunes del gen KRAS. Sin embargo, no es posible descartar la presencia otras mutaciones infrecuentes o hasta ahora no descritas, como en el caso del estudio de Egoavil y cols. ${ }^{14}$, donde se reportó una nueva mutación G13R (la glicina es remplazada por una arginina) en pacientes peruanos. Se requieren estudios de secuenciación del ADN tumoral en aquellos pacientes sin mutación detectada para abordar esta posibilidad.

Con respecto a la localización de las mutaciones en KRAS (colon derecho, colon izquierdo y recto) y las características clínicas e histopatológicas, no se observaron diferencias estadísticamente significativas con los pacientes sin mutación en KRAS (KRAS silvestre). Tampoco se observaron diferencias significativas entre mutaciones de KRAS y el género de los pacientes, tal como en otros estudios ${ }^{18}$.

Recientemente, otro miembro de la familia de proteínas RAS, NRAS ha cobrado gran importancia al observar que algunos CCR poseen mutaciones en los codones 2, 3 y 4 de manera muy similar a KRAS, produciendo también una mala respuesta a los anticuerpos monoclonales anti-EGFR. La frecuencia de mutaciones en NRAS en pacientes con CCR es alrededor de $6 \%$, e incluso se ha observado cercana a $10 \%$ en algunos países como Grecia y Rumania ${ }^{30-32}$, por lo que se ha agregado al estudio junto con KRAS antes de determinar la terapia.

Mutaciones en otros componentes de la red de señalización EGFR tales como PI3K o BRAF también pueden activar constitutivamente la vía y pueden dar cuenta de la falta de respuesta a terapias con anticuerpos monoclonales de una fracción de los pacientes con cáncer colorrectal metastásico que no presentan mutación en KRAS.

En resumen, este trabajo muestra el mayor estudio del estado mutacional del gen KRAS en pacientes chilenos con CCR realizado con una técnica altamente sensible y validada para estudios de mutaciones somáticas. Las frecuencias de mutación KRAS fueron levemente inferiores a las frecuencias reportadas en pacientes españoles, pero mayores a las reportadas por un estudio realizado en pacientes peruanos. También se observaron distribuciones similares de mutaciones específicas de KRAS con otros estudios. Los resultados de este estudio no encontraron diferencias estadísticamente significativas entre las mutaciones KRAS y características clínicas e histopatológicas del CCR pero no se puede descartar la presencia de otras mutaciones no descritas en población chilena o en otros componentes de la vía de señalización del EGFR y que son fundamentales de ser determinadas para elegir la terapia más efectiva al paciente.

\section{Referencias}

1. Malumbres M, Barbacid M. RAS oncogenes: the first 30 years. Nat Rev Cancer 2003; 3: 459-65.

2. Normanno N, Pinto C, Castiglione F, Bardelli A, Gambacorta M, Botti G, et al. KRAS mutations testing in colorectal carcinoma patients in Italy: from guidelines to external quality assessment. PLoS One 2011; 6: e29146.

3. Bos JL. RAS oncogenes in human cancer: a review. CanRes 1989; 49: 4682-9.

4. Neumann J, Zeindl-Eberhart E, Kirchner T, Jung A. Frequency and type of KRAS mutations in routine diagnostic analysis of metastatic colorectal cancer. Pathol Res Pract 2009; 205: 858-62.

5. Allegra CJ, Jessup JM, Somerfield MR, Hamilton SR, Hammond EH, Hayes DF, et al. American Society of Clinical Oncology provisional clinical opinion: testing for KRAS gene mutations in patients with metastatic colorectal carcinoma to predict response to anti-epidermal growth factor receptor monoclonal antibody therapy. J Clin Oncol 2009; 27: 2091-6.

6. Amado RG, Wolf M, Peeters M, Van Cutsem E, Siena S, 
Freeman DJ, et al. Wild-type KRAS is required for panitumumab efficacy in patients with metastatic colorectal cancer. J Clin Oncol 2008; 26: 1626-34.

7. Karapetis CS, Khambata-Ford S, Jonker DJ, O'Callaghan CJ, Tu D, Tebbutt NC, et al. Kras mutations and benefit from cetuximab in advanced colorectal cancer. $\mathrm{N}$ Engl J Med 2008; 359: 1757-65.

8. Roth AD, Tejpar S, Delorenzi M, Yan P, Fiocca R, Klingbiel $\mathrm{D}$, et al. Prognostic role of KRAS and BRAF in stage II and III resected colon cancer: results of the translational study on the PETACC-3, EORTC 40993, SAKK 60-00 trial. J Clin Oncol 2010; 28 (3): 466-74.

9. Berg M, Danielsen SA, Ahlquist T, Merok MA, Agesen $\mathrm{TH}$, Vatn $\mathrm{MH}$, et al. DNA sequence profiles of the colorectal cancer critical gene set KRAS-BRAF-PIK3CAPTEN-TP53 related to age at disease onset. PloS One 2010; 5: e13978.

10. Herreros-Villanueva M, Gómez-Manero N, Muniz P, García-Giron C, Coma del Corral MJ. PIK3CA mutations in KRAS and BRAF wild type colorectal cancer patients. A study of Spanish population. Mol Biol Rep 2011; 38: 1347-51.

11. Sylvester BE, Huo D, Khramtsov A, Zhang J, Smalling RV, Olugbile S, et al. Molecular analysis of colorectal tumors within a diverse patient cohort at a single institution. Clin Can Res 2012; 18: 350-9.

12. Balschun K, Haag J, Wenke AK, von Schonfels W, Schwarz NT, Rocken C. KRAS, NRAS, PIK3CA exon 20, and BRAF genotypes in synchronous and metachronous primary colorectal cancers diagnostic and therapeutic implications. J Mol Diagn 2011; 13: 436-45.

13. Ciardiello F, Tejpar S, Normanno N, Mercadante D, Teague T, Wohlschlegel B, et al. Uptake of KRAS mutation testing in patients with metastatic colorectal cancer in Europe, Latin America and Asia. Target Oncol 2011; 6: 133-45.

14. Egoavil CM, Montenegro P, Soto JL, Casanova L, Sánchez-Lihon J, Castillejo MI, et al. Clinically important molecular features of Peruvian colorectal tumours: high prevalence of DNA mismatch repair deficiency and low incidence of KRAS mutations. Pathol 2011; 43: 228-33.

15. Miller BA, Scoppa SM, Feuer EJ. Racial/ethnic patterns in lifetime and age-conditional risk estimates for selected cancers. Cancer 2006; 106: 670-82.

16. Zárate AJ, Alonso FT, Garmendia ML, López-Kostner F. Increasing crude and adjusted mortality rates for colorectal cancer in a developing South American country. Colorectal Dis 2013; 15: 47-51.

17. Center MM, Jemal A, Smith RA, Ward E. Worldwide variations in colorectal cancer. CA Cancer J Clin 2009; 59: 366-78.
18. Roa IST, Majlis A, Schalper K. Mutación del gen KRAS en el cáncer de colon y recto. Rev Med Chile 2013; 141: 1166-72.

19. Stefanoff CG, Hassan R, González AC, Andrade LA, Tabak DG, Romano S, et al. Laboratory strategies for efficient handling of paraffin-embedded tissues for molecular detection of clonality in non-hodgkin lymphomas. Diagn Mol Pathol 2003; 12: 79-87.

20. Knijn N, Mekenkamp LJ, Klomp M, Vink-Borger ME, Tol J, Teerenstra S, et al. KRAS mutation analysis: a comparison between primary tumours and matched liver metastases in 305 colorectal cancer patients. $\mathrm{Br} \mathrm{J}$ Cancer 2011; 104: 1020-6.

21. Winder T, Mundlein A, Rhomberg S, Dirschmid K, Hartmann BL, Knauer M, et al. Different types of K-Ras mutations are conversely associated with overall survival in patients with colorectal cancer. Oncol Rep 2009; 21 : 1283-7.

22. Herreros-Villanueva M, Rodrigo M, Claver M, Muniz P, Lastra E, García-Girón C, et al. KRAS, BRAF, EGFR and HER2 gene status in a Spanish population of colorectal cancer. Mol Biol Rep 2011; 38: 1315-20.

23. UNICEF. Atlas sociolingüístico de Pueblos Indígenas de América Latina. 2010.

24. Rocco P, Morales C, Moraga M, Miquel JF, Nervi F, Llop E, et al. Composición genética de la población chilena. Distribución de polimorfismos de DNA mitocondrial en grupos originarios y en la población mixta de Santiago. Rev Med Chile 2002; 130: 125-31.

25. Schubbert S, Shannon K, Bollag G. Hyperactive Ras in developmental disorders and cancer. Nat Rev Cancer 2007; 7: 295-308.

26. Loupakis F, Ruzzo A, Cremolini C, Vincenzi B, Salvatore L, Santini D, et al. KRAS codon 61, 146 and BRAF mutations predict resistance to cetuximab plus irinotecan in KRAS codon 12 and 13 wild-type metastatic colorectal cancer. Br J Cancer 2009; 13: 715-21.

27. De Roock W, Claes B, Bernasconi D, De Schutter J, Biesmans B, Fountzilas G, et al. Effects of KRAS, BRAF, NRAS, and PIK3CA mutations on the efficacy of cetuximab plus chemotherapy in chemotherapy-refractory metastatic colorectal cancer: a retrospective consortium analysis. Lancet Oncol 2010; 13: 753-62.

28. Janakiraman M, Vakiani E, Zeng Z, Pratilas CA, Taylor BS, Chitale D, et al. Genomic and biological characterization of exon 4 KRAS mutations in human cancer. Cancer Res 2010; 13: 5901-11.

29. Imamura $Y$, Lochhead P, Yamauchi M, Kuchiba A, Qian ZR, Liao X, et al. Analyses of clinicopathological, molecular, and prognostic associations of KRAS codon 61 and codon 146 mutations in colorectal cancer: 
cohort study and literature review. Mol Cancer 2014; 13: 135

30. Schirripa M, Cremolini C, Loupakis F, Morvillo M, Bergamo F, Zoratto F, et al. Role of NRAS mutations as prognostic and predictive markers in metastatic colorectal cancer. Int J Cancer 2014; doi: 10.1002/ijc.28955. [Epub ahead of print].

31. Negru S, Papadopoulou E, Apessos A, Stanculeanu DL,
Ciuleanu E, Volovat C, et al. KRAS, NRAS and BRAF mutations in Greek and Romanian patients with colorectal cancer: a cohort study. BMJ Open. 2014; 4 (5): e004652.

32. Meriggi F, Vermi W, Bertocchi P, Zaniboni A. The Emerging Role of NRAS Mutations in Colorectal Cancer Patients Selected for Anti-EGFR Therapies. Rev Recent Clin Trials. 2014. [Epub ahead of print]. 